Background In recent years there has been an increase of birth of premature babies and also in the increased survival of extremely premature with very low birth weight. The process of enabling these newborns requires multiple and complex care that are associated with significant ethical issues.

Objective To explore the experience of health professionals on ethical decision making in the care of the premature babies.

Methods We conducted a qualitative study of phenomenological nature, in the area of the constructivist paradigm, interviewing 13 doctors and 14 nurses who work in NICUs (Neonatal Intensive Care Units) located in the Centre region of Portugal.

Results On the deep discursive reflections, we understood the concerns and conflicts in difficult decision making, given the duty to revive in the limit of viability, the unquestionable respect to the human life and also the ethical duty and morally justified to limit or discontinue care, as response to the criterion of "best interests of the child". We underline some ambiguity surrounding the decision making to withhold treatment in situations of unfavourable prognostic, an understandable attitude ethically grounded in respect for life. They evidenced concerns about dying with dignity, yet there are no standards/recommendations for neonatal palliative care.

Conclusions We believe that reflection on practise could help to consolidate a culture of ethics on health professionals who work with premature infants and their parents.

We emphasise the importance of protocols and criteria that guide clinical practise regarding these situations.

\section{PL.92 MANAGEMENT OF MACROSOMIA IN NON DIABETIC WOMEN IN DISTRICT GENERAL HOSPITAL. IS INDUCTION OF LABOUR JUSTIFIED?}

doi:10.1136/archdischild-2013-303966.274

\section{A Yulia, F Shakur, M Matar. North Cumbria NHS Trust, Carlisle, UK}

Background Macrosomia refers to newborn with an excessive birth weight. Fetal macrosomia has been defined as birth weight of 4000 $4500 \mathrm{~g}$ or greater than $90 \%$ for gestational age after correcting for neonatal sex and ethnicity. Based on these definitions, macrosomia affects $1-10 \%$ of all pregnancies. Attempts at perinatal diagnosis of macrosomia have proven difficult and are often inaccurate. Thus, a diagnosis of fetal macrosomia can only be confirmed retrospectively.

Due to lack of data on management of macrosomia and associated perinatal outcomes in women without diabetes, we designed a retrospective study to examine the association between induction of labour (IOL) and mode of delivery (MOD) in low-risk women with neonatal macrosomia.

Methods Retrospective study was carried out over 1 year period (1 ${ }^{\text {st }}$ March $2011-29^{\text {th }}$ Feb 2012) looking at singleton births of macrosomic neonates from non-diabetic women at term. Outcome measures include MOD, 5 minutes Apgar, neonatal injury.

Results and Conclusions 232 women delivered neonates with birthweight of $>4000$ grammes. 18 women had elective CS, 90 women had spontaneous labour and 144 women were induced. 24 women were induced due to suspected macrosomia. Of all the 24 women who had IOL for suspected macrosomia, 38\% had emergency CS, 38\% had normal delivery and $25 \%$ had instrumental delivery. Higher rate of CS (38\% vs $8 \%$ ) was noted in women who were induced for suspected macrosomia compared to women who had spontaneous labour, and there is no significant difference in the 5 min Apgar and neonatal injury in the two groups.

\section{PL.93 CARING FOR THE PARTURIENT: VALORISATION OF CARE RECEIVED DURING LABOUR, DELIVERY AND POSTPARTUM}

doi:10.1136/archdischild-2013-303966.275

M Ferreira, P Nelas, G Aparício. Polytechnic Institute of Viseu - School Health - CIGDETS, Viseu, Portugal
Background Childbirth is a remarkable experience in the life of women and her family, so health professionals (obstetric doctor and nurse), should adopt a set of practises that promote healthy labour and birth, including respect for the physiological process and dynamic of each birth.

Objective To analyse the relationship between sociodemographic and obstetric variables with the valorisation of care received during labour and postpartum.

Method A quantitative, cross-sectional, descriptive and correlational study, consisted of 386 women interned in the obstetrics service of the Portuguese public hospitals who received a questionnaire consisting of sociodemographic variables, care provided, action before pain, social and familial support, attention to wellbeing and attention to postpartum/discharge.

Results The puerperal women were aged between 19 and 37 years, mostly married (79.5\%). 34.5\% have secondary education, $70.2 \%$ perform a work activity and $50 \%$ are primiparous. Parity, surveillance of pregnancy, preparation for childbirth and parenting time and hours in labour (this inversely), influence the importance of the care received in relation to the action before the pain and attention to the wellbeing.

Conclusion The orientation of obstetric care according to the woman allows for active participation in her birth plan, a sense of control, improves her self esteem and their level of satisfaction with the care received.

\section{PL.94 THE DECLINING RATE OF VBAC IN A DUBLIN TERTIARY CENTRE}

doi:10.1136/archdischild-2013-303966.276

M Farren, N Obaidi, A Mullally, CO Gorman, M Turner, N Farah. Coombe Women and Infants Universtiy Hospital, Dublin, Ireland

Promotion of VBAC has been an area of interest since the 1950's when the TOLAC trial emerged. We looked at rates in a tertiary referral centre, comparing the difference in VBAC rate among Para 1 (One previous LSCS) in 2011 and 2005. The results reveal a declining VBAC rate. The rate fell from $33.1 \%$ in 2005 to $23 \%$ in 2011

In $2011,56 \%$ had elective LSCS. Of those who laboured, $52 \%$ $(\mathrm{n}=166)$ had VBAC, 47.6\% $(\mathrm{n}=151)$ had Emergency LSCS. This was in comparison to 2005: $42 \%$ had Elective LSCS. Of those who laboured, 56.2\% ( $\mathrm{n}=189)$ had VBAC, $43.75 \%(\mathrm{n}=147)$ had Emergency LSCS.

\section{PL.95 OBSTETRIC PRACTISES DURING CHILDBIRTH: EXPECTATIONS, PERCEPTIONS AND SATISFACTION OF WOMEN}

doi:10.1136/archdischild-2013-303966.277

P Nelas, E Coutinho, C Chaves, J Duarte, B Arias. Institute Polytecnic of Viseu, Viseu, Portugal

Knowing what the woman expected to find and what she found in relation to obstetric practises, enables us to meet the needs, if we assess their satisfaction we will know if we can fill.

Objectives To determine if the expectations and satisfaction of women in relation to obstetric practises during the labour, delivery and postnatal influence their perception. To assess the expectations and satisfaction of women in relation to obstetric practises. Analyze the relationship between expectations and satisfaction.

Methods A quantitative, descriptive, analytic and correlational cross-sectional, with a sample of 405 mothers. The evaluation protocol includes the scale of importance and perception of Obstetric Practice (Roman Espinaco, Garrido Oliver, 2008) and the Questionnaire Experience and Satisfaction with Childbirth (Costa el al, 2004). 